\title{
Ictal-Interictal Continuum: When to Worry About the Continuous Electroencephalography Pattern
}

\author{
Justine Cormier, MD ${ }^{1}$ Carolina B. Maciel, MD ${ }^{1,2}$ Emily J. Gilmore, MD, MS
}

${ }^{1}$ Department of Neurology, Yale University School of Medicine, New Haven, Connecticut

2 Department of Neurology, UF Health Shands Hospital, University of Florida College of Medicine, Gainesville, Florida

${ }^{3}$ Division of Neurocritical Care and Emergency Neurology, Department of Neurology, Yale University School of Medicine, New Haven, Connecticut

Semin Respir Crit Care Med 2017;38:793-806.

\author{
Address for correspondence Emily J. Gilmore, MD, MS, Department of \\ Neurology, Yale University School of Medicine and Yale-New Haven \\ Hospital, 15 York Street, Building LCI, 10th floor, Room 1000B, New \\ Haven, CT 06520 (e-mail: emily.gilmore@yale.edu).
}

\begin{abstract}
Keywords

- ictal-interictal continuum

- periodic discharges

- rhythmic delta activity

- nonconvulsive status epilepticus

- continuous electroencephalography

Continuous electroencephalography (CEEG) monitoring is an invaluable tool in the evaluation of encephalopathy and coma in critically ill patients. Marked increases in cEEG monitoring, coinciding with several societal guideline statements in the last decade, have allowed earlier detection and treatment of clearly harmful patterns, including nonconvulsive seizures (NCSz) and nonconvulsive status epilepticus (NCSE). However, it has also unmasked a range of EEG patterns of less clear clinical significance, with some more "malignant" than others given their potential association with increased neuronal stress and secondary brain injury. These patterns lay on a spectrum often referred to as the ictal-interictal continuum (IIC). To date, no definitive guidelines exist for the management of these potentially harmful EEG patterns, thus presenting a clinical dilemma for critical care physicians. Here, we review the various IIC patterns, their associated features, seizure risk, and outcomes and also propose a clinical approach to management based on the available data and expert opinion.
\end{abstract}

A 58-year-old woman with longstanding depression, anxiety, and chronic back pain on baclofen and codeine was admitted to an outside hospital with an acute change in mental status. Earlier in the day, she was noted to be confused following a fall and became unresponsive during transport to the hospital. On arrival to the emergency department, she was noted to be afebrile and hemodynamically stable, but hypoxemic to $90 \%$ on room air and comatose without lateralizing signs on examination. Her initial laboratory studies demonstrated significant metabolic derangements, which included elevated creatinine kinase $(1,240 \mathrm{U} / \mathrm{L})$, acute kidney injury (creatinine: $2.2 \mathrm{mg} / \mathrm{dL}$, serum urea nitrogen: $59 \mathrm{mg} / \mathrm{dL}$ ), transaminitis (alanine aminotransferase: $390 \mathrm{U} / \mathrm{L}$, aspartate aminotransferase: $565 \mathrm{U} / \mathrm{L}$ ), hyperammonemia (ammonia: $52 \mu \mathrm{mol} / \mathrm{L}$ ), and leukocytosis with left shift (white blood cell:
18,800 with $23 \%$ bands). A urine toxicological screen was positive for opioids and acetaminophen. Also, she was estimated to have ingested at least $210 \mathrm{mg}$ of baclofen, based on a pill count. Computed tomography without contrast of the brain demonstrated only a nasal bone fracture. She was presumed to have overdosed with opioids, acetaminophen, and baclofen, and received supportive therapy and N-acetylcysteine. However, she continued to deteriorate over 3 days with worsening multiorgan failure in the setting of severe sepsis secondary to perforated sigmoid colitis. A routine electroencephalography (EEG) reportedly demonstrated epileptiform discharges triggering a transfer to our institution for long-term monitoring and continued care. Brain magnetic resonance imaging (MRI) was motion degraded but otherwise unremarkable. Continuous EEG (cEEG)
Issue Theme Advancements in Neurocritical Care and Emergency Neurology; Guest Editors: David Y. Hwang, MD, FNCS, and David M. Greer, MD, MA, FCCM, FAHA, FNCS, FAAN, FANA
Copyright (C) 2017 by Thieme Medical Publishers, Inc., 333 Seventh Avenue, New York, NY 10001, USA. Tel: +1(212) 584-4662.
DOI https://doi.org/ 10.1055/s-0037-1607987. ISSN 1069-3424. 
demonstrated nonconvulsive status epilepticus (NCSE) with generalized highly epileptiform bursts comprising greater than $50 \%$ of the recording (-Fig. 1A, B). Clinically, she remained comatose with intermittent, random, nonsynchro- nous jerks of her extremities along with stereotypic bilateral leg adduction, neither of which consistently occurred timelocked with epileptiform activity on EEG. It is important to note that the etiology of the NCSE, though likely

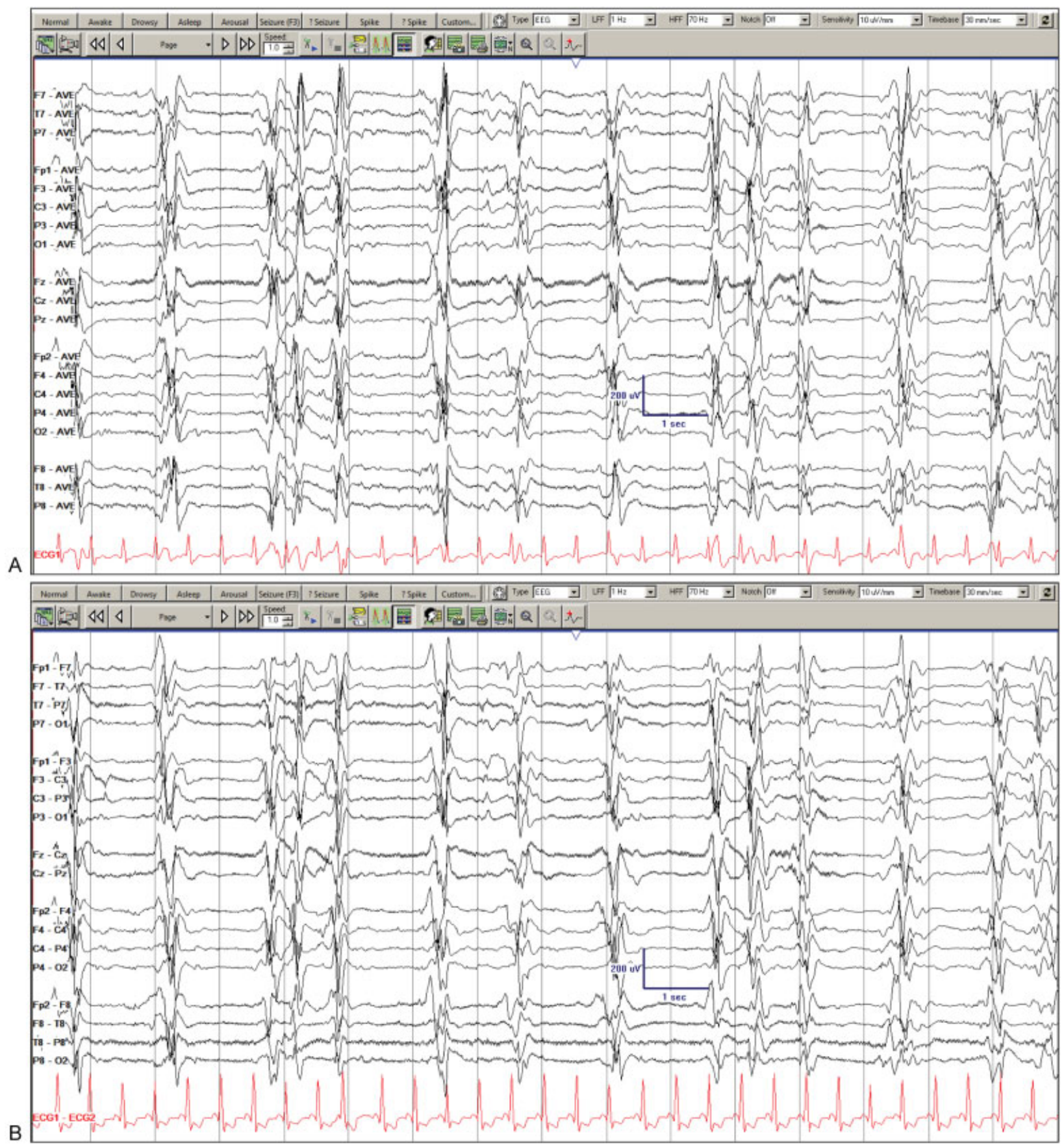

Fig. 1 Electroencephalographic evolution from nonconvulsive status epilepticus and continued diffuse hyperexcitability with ictal-interictal continuum following treatment. All epochs demonstrate at least 15 seconds of recording captured with high-pass filter at $1 \mathrm{~Hz}$, low-pass filter at $70 \mathrm{~Hz}$, and paper speed of $30 \mathrm{~mm} / \mathrm{s}$. Sensitivity is set at $7 \mu \mathrm{V} / \mathrm{mm}$ and notch filter "off," unless otherwise specified. (A) Common average reference montage showing generalized highly epileptiform bursts of polyspikes and spikes shifting maxima in a burst suppressed background. Note the high amplitude of epileptiform activity reaching 200 to $300 \mu \mathrm{V}$ and the need to adjust gain (dialed down at $10 \mu \mathrm{V} / \mathrm{mm}$ ) to allow for better characterization of morphology of discharges. (B) Same epoch and settings displayed on longitudinal bipolar anatomical montage. (C) Resolution of nonconvulsive status epilepticus and overall decrease in the ictal appearance of discharges with decreased sharpness and amplitude components of bursts. There is also improved continuity of background, now transitioned from burst suppression to a discontinuous recording. (D) Ictal-interictal continuum with bursts of irregular, sharply contoured, 3 to $6 \mathrm{~Hz}$ waveforms lasting 1 to 3 seconds admixed with fast activity displayed in longitudinal bipolar anatomical montage; Notch filter "off," note the $60 \mathrm{~Hz}$ artifact on midline leads. (E) Same epoch and montage with Notch filter "on" and resolution of $60 \mathrm{~Hz}$ artifact. (F) Continuous background consisting of rich frequencies admixed with poorly formed sharply contoured generalized discharges. 


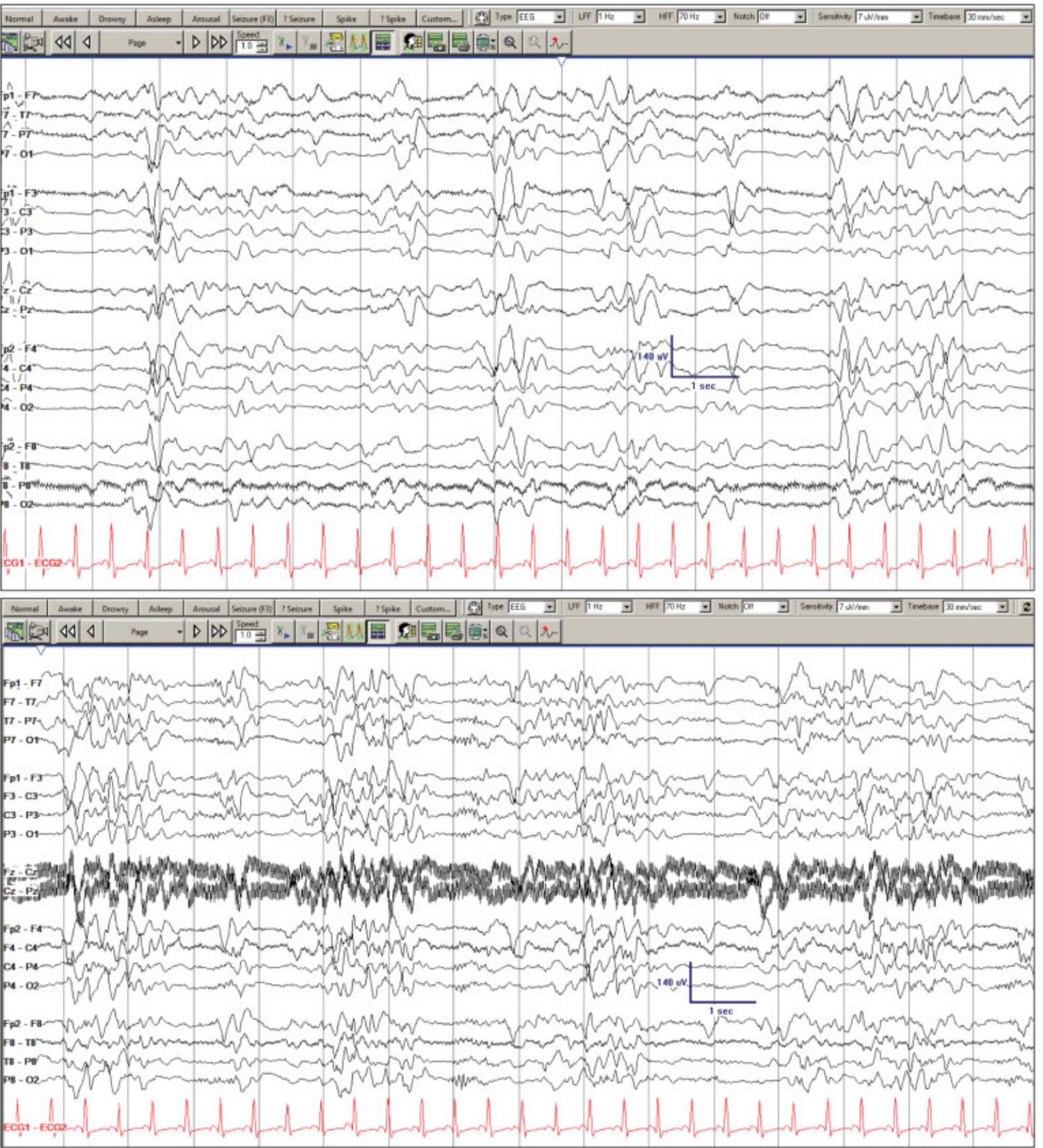

Fig. 1 (Continued)

multifactorial, was predominantly due to toxic-metabolic derangements in the setting of baclofen toxicity, liver and renal failure, and sepsis. While there is controversy over the most appropriate treatment of NCSE triggered by metabolic derangements, it is reasonable to follow the available guidelines for the treatment of status epilepticus. She was loaded with $2,000 \mathrm{mg}$ of levetiracetam and received two $4 \mathrm{mg}$ boluses of lorazepam. This resulted in a reduction of the ictal burden on EEG such that it no longer met the criteria for NCSE ( $<30 \mathrm{~min} / \mathrm{h}$ of recording; - Fig. 1C), but there was no improvement in her neurological examination. A $20 \mathrm{mg} / \mathrm{kg}$ load of fosphenytoin failed to demonstrate further ictal burden reduction despite a free level of $3.4 \mu \mathrm{g} / \mathrm{mL}$. She was then started on continuous midazolam infusion and titrated to $30 \mathrm{mg} / \mathrm{h}$, which resulted in burst suppression with frequent generalized periodic discharges. The decision to use an anesthetic infusion was based on the refractory nature of the electrographic patterns to other antiseizure medications as well as the high likelihood of a more prolonged course and slow resolution of offending metabolic derangements given the severity of the infection, multiorgan failure, and delayed clearance of baclofen. On the following day, lacosamide (load of $300 \mathrm{mg}$ followed by $75 \mathrm{mg}$ every 8 hours) was added to levetiracetam (renally dosed at $500 \mathrm{mg}$ every 6 hours) in 


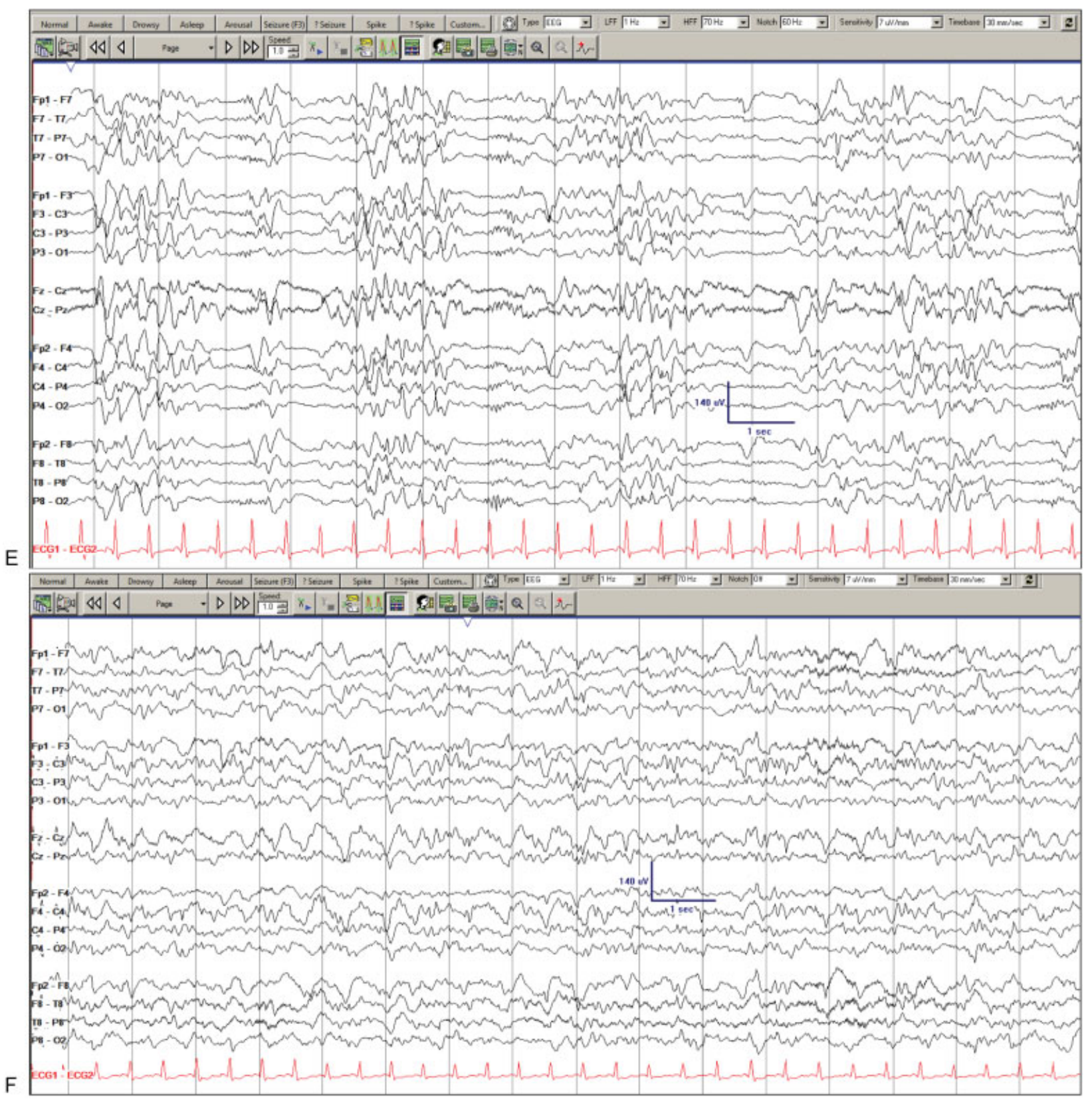

Fig. 1 (Continued)

anticipation of weaning midazolam, which was completed within 12 hours. In this setting, the emergence of abundant bursts of sharply contoured theta and delta discharges lasting 1 to 3 seconds were captured comprising 30\% of the recording and considered to lie on the IIC ( - Fig. 1D, E). At that point, it would have been reasonable to either pursue careful monitoring on CEEG or to continue aggressive pharmacologic treatment to decrease the IIC pattern burden further. A low-dose lorazepam taper was chosen as a bridging strategy, starting at $2 \mathrm{mg}$ every 6 hours and subsequently weaned off over 4 days (-Fig. 1F). There was concurrent gradual electrographic and clinical improvement with resolution of the IIC pattern in the context of resolving metabolic disarray and treatment of her infection. After 10 days of the initial recording that had demonstrated
NCSE, a repeat 60-minute EEG was normal (-Fig. 2A, B). She was discharged on lacosamide and levetiracetam, which were weaned off as an outpatient. Since her NCSE was provoked by toxic, metabolic, infectious disarray, she does not have epilepsy and does not necessarily warrant lifelong antiseizure medication.

\section{Background}

cEEG monitoring is becoming a ubiquitous tool in the evaluation, management, and prognostication of encephalopathy and coma in critically ill patients. Historically, there has been wide practice variation pertaining to indications for and duration of cEEG monitoring, in large part due to limited resources and lack of established guidelines. ${ }^{1}$ However, in the 


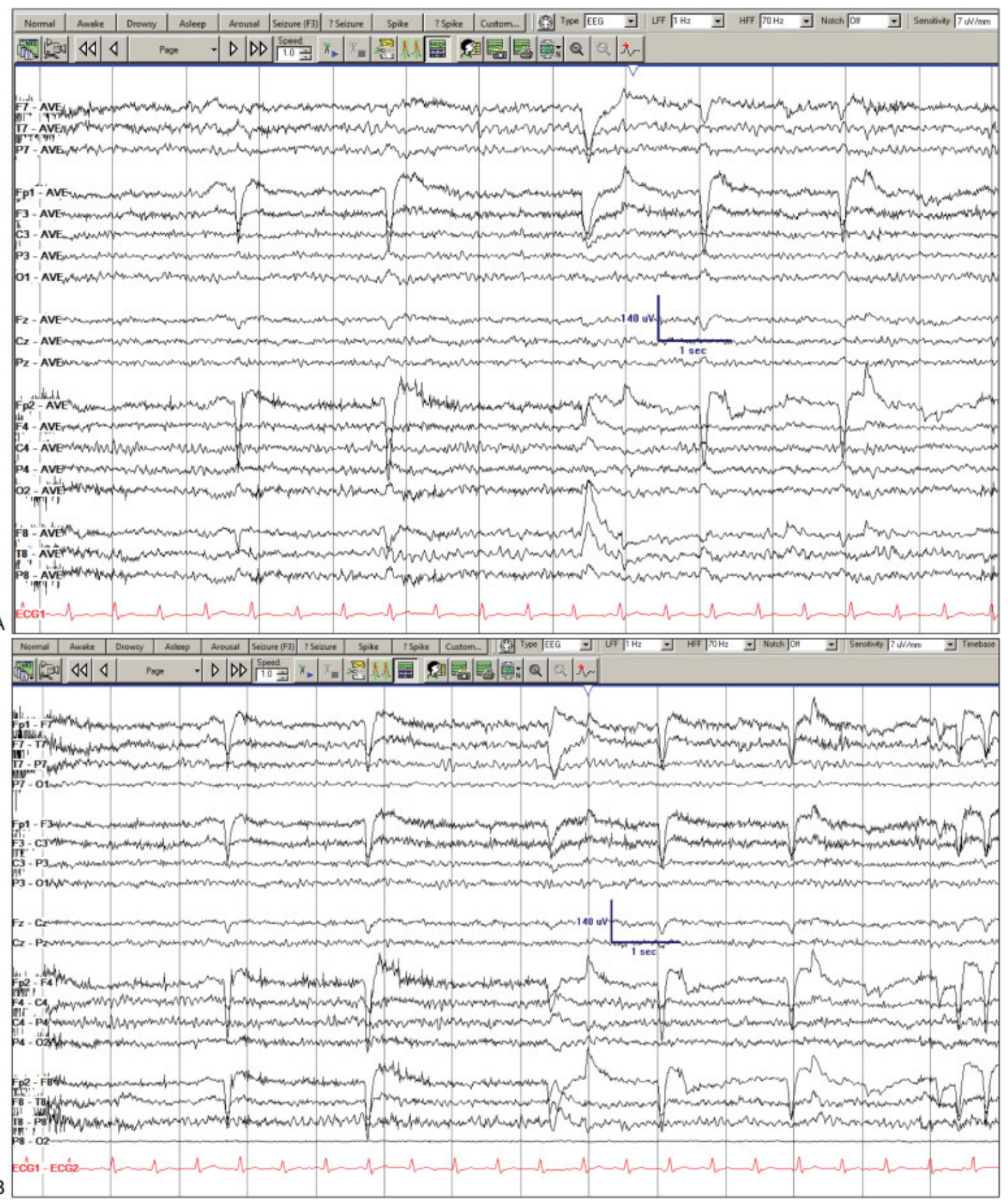

Fig. 2 Repeat 60 minutes electroencephalogram 10 days later. All epochs demonstrate at least 15 seconds of recording captured with a highpass filter at $1 \mathrm{~Hz}$, low-pass filter at $70 \mathrm{~Hz}$, and paper speed of $30 \mathrm{~mm} / \mathrm{s}$. Sensitivity is set at $7 \mu \mathrm{V} / \mathrm{mm}$ and notch filter "off," unless otherwise specified. (A) Common average reference montage showing a normal awake background. (B) Same epoch and settings displayed on longitudinal bipolar anatomical montage. Note that the isoelectric P8-O2 channel is a result of dipole cancellation from the proximity of leads on right posterior region.

last 5 years, several national and international societies, including the Neurocritical Care Society, American Clinical Neurophysiology Society (ACNS), and the European Society of Intensive Care Medicine, have published clear guidelines for utilization of $\mathrm{CEEG}$ in the critical care setting. ${ }^{2-4}$ Combined, these guidelines strongly recommend the use of cEEG to: (1) detect and aid in the management of nonconvulsive seizures (NCSz) and NCSE in the setting of persistent, unexplained alterations in mental status, (2) aid in neuroprognostication after cardiac arrest, (3) identify electrographic seizures in 
patients with acute stroke, traumatic brain injury, or fluctuating mental status without known brain injury (e.g., in sepsisassociated encephalopathy); (4) determine degree of encephalopathy in patients receiving intravenous (IV) sedation or undergoing pharmacologically induced coma; and (5) assess for seizure activity in high-risk patients requiring pharmacological paralysis, such as with therapeutic hypothermia and extracorporeal membrane oxygenation (ECMO). ${ }^{2,3}$ While the increased use of cEEG has allowed earlier detection of NCSz and NCSE, both of which have been shown to be highly associated with poor outcomes and increased mortality, ${ }^{5-12}$ it has also led to an increased detection of epileptiform patterns-periodic discharges and rhythmic delta activitywhich are of less clear clinical significance. These findings are abnormal and share some features of ictal rhythms, thus tempting clinicians treating with benzodiazepines or other antiseizure medications, which are not without risks. Although these patterns do not fully meet criteria for electrographic seizures, ${ }^{13}$ it remains unclear not only if they cause a similar degree of neuronal injury or worse outcomes, but also whether they warrant the same degree of aggressive treatment as definitive seizures. Given this uncertainty, these patterns are felt to exist on a spectrum, with more malignant patterns at the ictal end. This is referred to as the IIC (-Fig. 3). ${ }^{14}$

The ACNS has created a standardized set of critical care EEG terminology to assist with the identification and classification of these abnormal patterns and to foster research by creating a uniform nomenclature. ${ }^{13}$ This terminology has been widely accepted and shown to have high interrater reliability for most terms, ${ }^{15}$ although the ability to identify triphasic wave (TW) morphology and spatiotemporal evolu- tion of EEG patterns remains a challenge among clinicians. ${ }^{15,16}$ In addition to the ACNS criteria, the Salzburg criteria were proposed as a standardized set of guidelines to reliably identify NCSE by EEG, with the more recent modified Salzburg criteria updated to include ACNS terminology, resulting in increased specificity. ${ }^{17,18}$ According to these criteria, to be considered NCSE, at least one of the following criteria must be met and be continuously present for at least 10 seconds: (1) epileptiform patterns occurring at $>2.5 \mathrm{~Hz}$; (2) subtle concurrent clinical phenomena; or (3) typical spatiotemporal evolution. ${ }^{18}$

There is no such set of uniform guidelines with unequivocal recommendations addressing the management of IIC patterns, and thus the appropriate acute and long-term treatment remain a challenge for the critical care clinician. Here, we will review EEG patterns that exist on the IIC-their characteristics, typical etiologies, associated seizure risk, and outcomes-and propose a therapeutic approach based on the available data and expert opinion.

\section{Periodic Discharges}

Periodic discharges (PDs) are waveforms lasting $\leq 0.5$ seconds, consisting of not more than three phases with a relatively uniform morphology, duration, and interdischarge interval. ${ }^{13}$ PDs can be further classified by region of onset as lateralized, generalized, or bilateral independent. Regardless of localization, PDs found on the IIC are often between 1 and $2.9 \mathrm{~Hz}$ and associated with "plus" features, that is, features that render a more ictal connotation to these patterns. These features include superimposed fast (" + F") or rhythmic delta activity (" $+\mathrm{R}$ "). ${ }^{13,19}$ It is also possible to have both

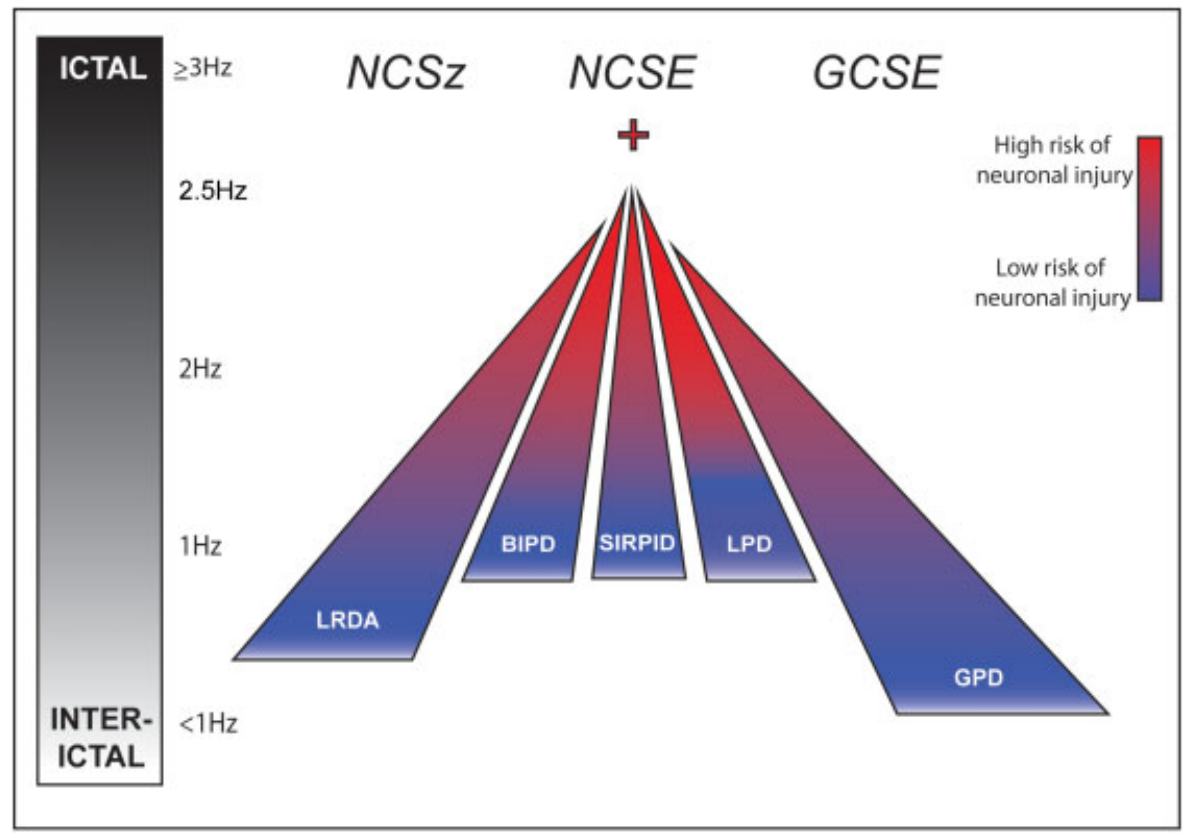

Fig. 3 This figure demonstrates the various electroencephalogram patterns felt to lay on the IIC. The frequency of discharges is shown on the yaxis. The color refers to the likelihood of causing secondary neuronal injury, with red being more harmful and blue being less harmful. Patterns on the IIC occur at frequencies $<3 \mathrm{~Hz}$, with patterns $\geq 3 \mathrm{~Hz}$ felt to be unequivocally ictal (including NCSE, NCSz, and GCSE). The plus sign refers to plus features that may render patterns closer to the ictal end of the continuum. GCSE, generalized convulsive status epilepticus; NCSE, nonconvulsive status epilepticus; NCSz, nonconvulsive seizures. 
superimposed fast and rhythmic activity, denoted as “ + FR." ${ }^{13}$ When PDs are of a faster frequency and associated with plus features, they lay closer to the ictal end of the IIC and are associated with higher risk of seizures in patients with acute structural lesions (see - Fig. 3)..$^{20}$

\section{Lateralized Periodic Discharges}

Historically referred to as periodic lateralized epileptiform discharges, the newly termed lateralized PDs (LPDs) are uniform, repetitive PDs with a clear lateral predominance. These discharges often have a sharp or spike morphology and are typically 100 to $300 \mu \mathrm{V}$ in amplitude. ${ }^{13}$ LPDs are uncommon in the general population, with reports ranging from 0.4 to $1 \%$ of pooled patients with a broad range of pathologies, chronicity, and indication for monitoring including those undergoing outpatient EEG. ${ }^{21,22}$ They are, however, the most commonly observed periodic pattern in critically ill patients, seen in 6.1 to $8.6 \%$ of hospitalized patients. $^{23-26}$

\section{When Do We See Them?}

LPDs are most often seen in structural brain injury, either acute or chronic, ${ }^{27}$ and either cortical or subcortical. ${ }^{28}$ Acute stroke is the most commonly reported etiology, ${ }^{11,29-31}$ though LPDs are also seen in patients with central nervous system (CNS) tumors or mass lesions, encephalitis, CNS infection, traumatic brain injury, and hemorrhage, ${ }^{11,20-22,27,31-33}$ reported in up to $13 \%$ of patients with intracerebral hemorrhage (ICH). ${ }^{32}$ LPDs typically occur ipsilateral to the injury, and in acute stroke, data are suggesting that LPDs originate specifically from the ischemic penumbra rather than the infarcted core tissue. ${ }^{34}$ These data support the hypothesis that LPDs represent an acute, partial, and transient dysfunction in a specific brain area; ${ }^{33}$ however, LPDs have also been well described in chronic and static lesions as well as epilepsy. ${ }^{35-37}$ Patients with LPDs often have a corresponding focal abnormality on neurological examination contralateral to the origin of the LPDs, ${ }^{27,38}$ though it is nearly impossible to determine if this clinical correlate is attributable to LPDs or the underlying structural lesion.

LPDs can also occur in the setting of systemic infection and toxic or metabolic insults, even in the absence of a structural brain lesion. ${ }^{33}$ There has been increasing interest in cEEG monitoring of patients in the nonneurological intensive care setting who present with sepsis and altered mental status and do not have an acute neurological injury. Multiple studies have shown that 1 in 10 septic patients admitted to the medical intensive care unit undergoing monitoring will have NCSz captured on cEEG, and up to one-quarter (17-25\%) of them have PDs. ${ }^{39,40}$ A study of patients with sepsis in the surgical intensive care unit found even higher rates of both NCSz (16\%) and PDs (29\%). ${ }^{41}$ Although this cohort included patients with acute brain injury (12\% of patients), there was no statistically significant association between acute brain injury and rate of NCSz or PDs. ${ }^{41}$ Other studies, however, have suggested that the patients most at risk for LPDs are those with both a focal brain injury and concomitant toxic, metabolic, or infectious processes. ${ }^{22}$

\section{Should We Worry?}

LPDs are highly associated with increased risk of seizures. Numerous studies have shown this to be the case, with reports of electrographic seizures in 40 to 95\% of inpatients with LPDs on cEEG. ${ }^{11,20,26,27,30,31,33}$ As with other patterns discussed here, when LPDs are associated with plus features, their correlation with clinical and nonconvulsive seizures and with NCSE increases. ${ }^{20,22,42}$ Reported rates of electrographic seizures and status epilepticus can reach $100 \%$ in patients with LPD associated with plus features. ${ }^{22}$ One study demonstrating increased seizure risk in patients with LPDs plus over LPDs without associated features (odds ratio [OR] of 2) also found greater risk of seizures with higher frequency LPDs $(>2 \mathrm{~Hz}){ }^{43}$ Despite their correlation with seizures, LPDs can also be seen in patients who do not go on to have clinical or electrographic seizures in up to $50 \%$ of cases, and predicting which subsets of patients are at lower risk is nearly impossible. ${ }^{33}$ In addition to the increased risk of seizures, recent work has shown that LPDs may be predictive of delayed cerebral ischemia in patients with aneurysmal subarachnoid hemorrhage (aSAH), although it is unclear if they are mechanistically involved, predictive markers, or merely disease bystanders. ${ }^{44}$

While the association between LPDs and seizure risk is well-accepted, a more controversial theory is that LPDs may themselves be an ictal phenomenon. Some groups argue that LPDs are considered ictal in cases where stereotypic focal movements-such as in focal motor seizures or epilepsia partialis continua-are time-locked to discharges. ${ }^{22,30}$ Others have argued that even LPDs associated with nonmotor clinical signs, such as aphasia or confusional states, ${ }^{45-48}$ can be considered ictal, particularly if there is clinical and electrographic improvement with anti seizure drug treatment. ${ }^{24}$

Ictal or interictal, LPDs are unequivocally associated with high morbidity and mortality. They have been shown to be independently associated with increased rates of severe disability, vegetative state, and death, with mortality rates ranging from 25 to $41 \%{ }^{22,27,30,31,41,49}$ Studies have also shown lower likelihood of being discharged to home (OR: $0.2)^{49}$ and poor functional outcomes at discharge, with one study reporting only $21 \%$ of 82 patients with LPDs during admission being functionally independent 1 year after hospital discharge. ${ }^{30,50}$ Many patients with LPDs on cEEG go on to have seizures well after hospital discharge, with reports ranging from 10 to $60 \%{ }^{10,30,33}$

\section{Bilateral Independent Periodic Discharges}

Bilateral independent PDs (BIPDs) are much less prevalent than LPDs, typically reported in less than $1 \%$ of patients undergoing cEEG. ${ }^{22,23,27}$ They are repetitive discharges that occur independently (asynchronously) between hemispheres, but similar to LPDs, have regular or nearly regular interdischarge intervals up to $3 \mathrm{~Hz} .{ }^{13}$

\section{When Do We See Them?}

BIPDs are typically found in acute and subacute brain injury, including CNS infection, ${ }^{38,51}$ anoxic brain injury, ${ }^{38}$ tumors, ${ }^{27}$ strokes, ${ }^{27}$ and metabolic disturbances. ${ }^{52}$ They are more rarely seen in $\mathrm{ICH}^{32}$ 


\section{Should We Worry?}

Although less commonly seen than LPDs, BIPDs are also highly associated with seizures and carry an even worse prognosis. Seizures are recorded in 43 to $78 \%$ of patients with BIPDs, ${ }^{22,27,38}$ although rates approached $100 \%$ in a small case series of patients with CNS infection ${ }^{51}$ and another series of four patients with BIPDs of varying etiologies. ${ }^{52}$ Patients with BIPDs on cEEG are more likely to have generalized seizures than focal seizures, and are more likely to be comatose on examination than those without BIPDs. ${ }^{38}$ Mortality rates are also astonishingly high, ranging from 39 to $100 \% .^{22,27,38,52}$ Similar to LPDs, patients with BIPDs who do survive to hospital discharge have poor outcomes and low likelihood of functional independence at 1 year. ${ }^{27,52}$

\section{Generalized Periodic Discharges}

Generalized PDs (GPDs) are repetitive, synchronous discharges occurring in both hemispheres at regular intervals with a clear interdischarge interval. ${ }^{13,53}$ They may co-occur with LPDs, but reports of their prevalence vary, ranging from 0.8 to $1.8 \%$ in some studies, ${ }^{23,25}$ and up to $4.5 \%$ in one review of 3,064 patients undergoing CEEG. $^{53}$

\section{When Do We See Them?}

GPDs are most commonly associated with toxic-metabolic disturbances and sepsis, even more so than LPDs and BIPDs. ${ }^{27,53-55}$ They are often seen in acute brain injury as well, with stroke and hypoxic-ischemic injury (HIE) being among the most common etiologies. ${ }^{27,29,53,56}$ They are less commonly seen in ICH and traumatic brain injury (TBI). ${ }^{32,53}$ While LPDs are more often associated with focal neurological deficits, ${ }^{27,33}$ GPDs are often seen with severe encephalopathy or coma, ${ }^{27}$ reflecting a more diffuse process and consistent with an increased incidence of GPDs with global insults.

\section{Should We Worry?}

Similar to LPDs and BIPDs, though to a lesser degree, GPDs have been shown to be associated with seizures and particularly so with NCSz and NCSE. ${ }^{25,27,53}$ In a study of 3,064 patients undergoing cEEG, GPDs were associated with NCSz in $26 \%$ (vs. $7.5 \%$ in controls) and with NCSE in $21.5 \%$ (vs. $6.5 \%$ in controls). More recently, a large series of 4,772 critically ill patients demonstrated what has been seen with all PDs-that more complex discharges with "plus" features and higher frequencies $(>1.5 \mathrm{~Hz})$ portend an even higher risk of seizures. ${ }^{43}$

Many studies have reported significantly increased morbidity and mortality in patients with GPDs, ${ }^{27,53}$ and this is particularly the case in patients with HIE or TBI. ${ }^{29,56}$ One study showed no evidence of worse outcomes in patients with GPDs when controlling for age, etiology, and level of consciousness, suggesting that GPDs may, perhaps, be disease bystanders. ${ }^{53}$ It has also been shown that patients with potentially reversible toxic-metabolic causes may have better outcomes. ${ }^{55}$

\section{What about Triphasic Waves?}

TWs are a modifier to PDs that further characterize the morphology of the waveform. They consist of three phases, each of longer duration than the preceding wave and consisting of a surface positive wave $>70 \mu \mathrm{V}$ both preceded and followed by a negative surface wave of smaller amplitude (-Fig. 4). ${ }^{13,57}$ First described by Foley et al in 1950, they were historically felt to be primarily associated with hepatic encephalopathy and not thought to portend a higher seizure risk. ${ }^{16}$ However, since that time, TWs have been shown to be present in a wide variety of toxic-metabolic disturbances, including hyponatremia, hypothyroid states, sepsis, lithium toxicity, and hypertensive encephalopathy, ${ }^{58,59}$ and may represent a combination of structural brain lesion and metabolic disturbance. ${ }^{57,59}$ Furthermore, multiple studies have now shown that TW have a similar risk of seizures as other PDs. ${ }^{16,57}$

\section{Rhythmic Delta Activity}

Rhythmic delta activity (RDA) consists of waveforms of relatively uniform duration and morphology that occur without an interval between consecutive waveforms. ${ }^{13}$ They can be lateralized (LRDA) or generalized (GRDA), although the RDA typically felt to lay on the IIC is lateralized, and like PDs, is often associated with increased frequency and plus features that signify a higher likelihood of seizures and worse outcomes (see - Fig. 3). These additional features include superimposed fast activity (" $+F$ "), frequent intermixed sharp waves or spikes (“+ S"), or both (“+ FS”). ${ }^{13,19}$

\section{Lateralized Rhythmic Delta Activity}

LRDA refers to a unilateral or bilateral synchronous, but an asymmetric pattern. ${ }^{13,26}$ In a study of 558 acutely ill patients monitored with cEEG, $4.7 \%$ had LRDA, and in $44 \%$ of these patients, LRDA co-occurred with LPDs. ${ }^{26}$

\section{When Do We See It?}

As with many of the epileptiform patterns described in this review, LRDA is seen in a variety of acute and remote focal CNS lesions; however, one study suggests that it is most commonly observed in intracerebral and subarachnoid hemorrhages. $^{26}$ of note, of the 27 patients with LRDA in this study, 70\% were found to have a focal abnormality on neurological examination that correlated with the laterality of the observed LRDA. ${ }^{26}$

\section{Should We Worry?}

Similar to LPDs, LRDA has been shown to be highly associated with seizures, and NCSz in particular. ${ }^{26}$ Of the $4.7 \%$ of patients with LRDA in the study mentioned above, acute seizures were seen in $53 \%$ of patients with frontal LRDA and $80 \%$ of patients with nonfrontal LRDA. ${ }^{26}$ In another extensive study of 4,772 patients undergoing CEEG, LRDA was associated with seizures in 25 to $44 \%$ of patients, and portended a much higher seizure risk when observed at a frequency of $1.5 \mathrm{~Hz}$ or greater or when associated with a plus modifier (OR: 1.8). ${ }^{43}$

\section{Stimulus-Induced Rhythmic, Periodic or Ictal Discharges}

Another phenomenon commonly seen on EEG recordings of critically ill patients consists of hyperexcitable discharges 


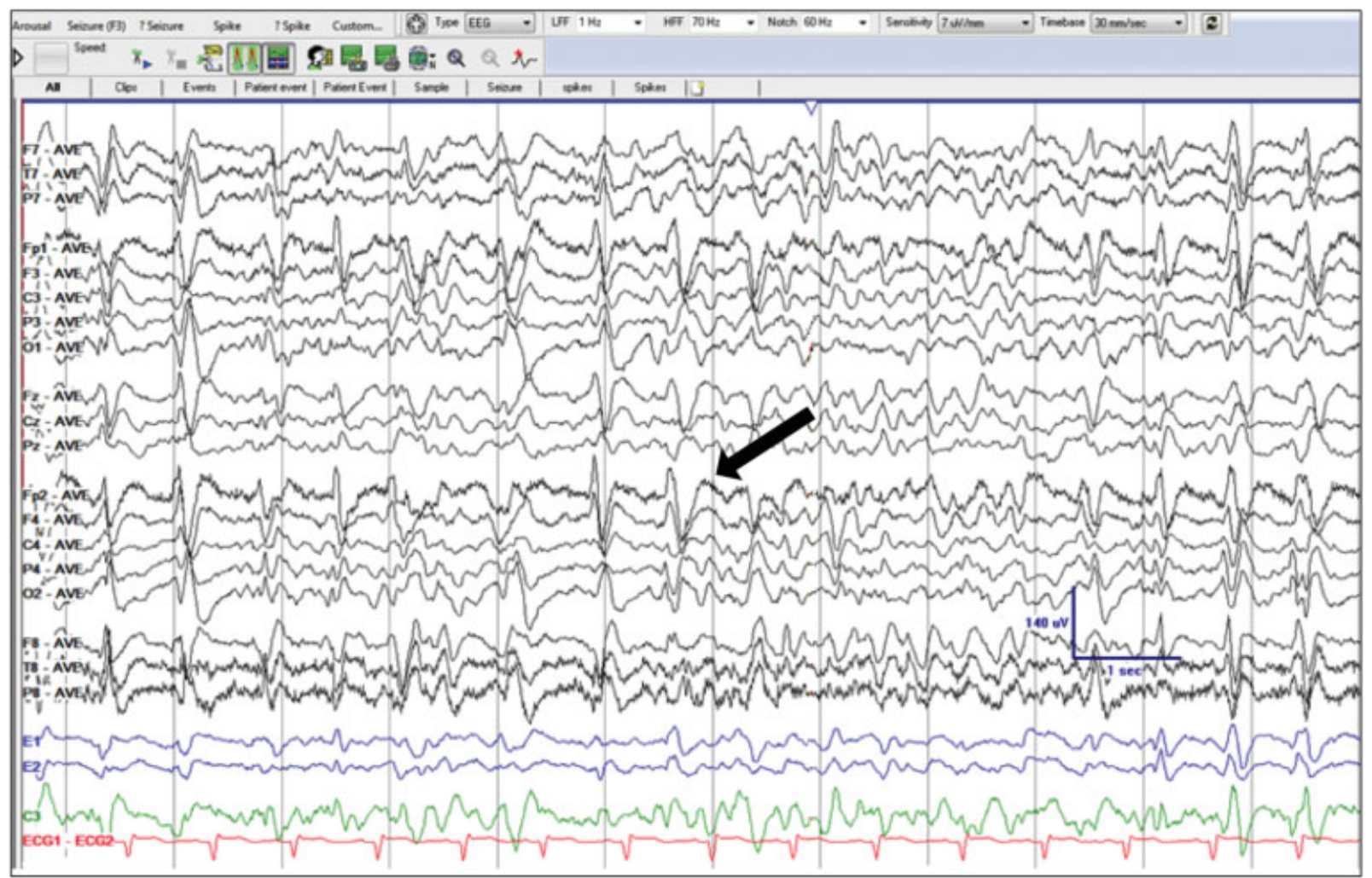

Fig. 4 Generalized periodic discharges with triphasic morphology. This epoch demonstrates 12 seconds of recording captured with a high-pass filter at $1 \mathrm{~Hz}$, a low-pass filter at $70 \mathrm{~Hz}$, the paper speed of $30 \mathrm{~mm} / \mathrm{s}$, sensitivity at $7 \mu \mathrm{V} / \mathrm{mm}$, and notch filter "on" in a common average referential montage. The black arrow points toward a discharge with a typical triphasic morphology.

that are consistently elicited by stimulation (e.g., suctioning, turning, bedside nursing care). ${ }^{19,60}$ Reports have shown stimulus-induced rhythmic, periodic or ictal discharges (SIRPIDs) to be present in 10 to $34 \%$ of hospitalized patients being monitored on cEEG. ${ }^{60-62}$

\section{When Do We See Them?}

They are most commonly seen in patients with acute brain injury; however, they have been reported in a wide range of conditions including stroke, ICH, subarachnoid hemorrhage (SAH), TBI, HIE, status epilepticus, and neurodegenerative disorders, such as Creutzfeldt-Jakob disease, as well as more systemic conditions, such as drug toxicity, hyponatremia, and other metabolic derangements. ${ }^{60,63-65}$

\section{Should We Worry?}

While commonly seen in critically ill patients, the significance of SIRPIDs is uncertain. Some studies seem to suggest a strong association between SIRPIDs and seizures given their frequent co-occurrence in critically ill patients, and particularly those with acute brain injury; ${ }^{60,61,64}$ however, it would appear that this association is specific to NCSE and that there is no association between SIRPIDs and seizures out of the context of status epilepticus. ${ }^{60,64}$ As with LPDs, there is some debate as to whether SIRPIDs may themselves be ictal phenomena. Some would suggest that this is possible given reports of both clinical and electrographic improvement with benzodiazepines. ${ }^{66}$ Still, others argue that these pat- terns are not ictal given a few case reports showing absence of cerebral hyperperfusion during SIRPIDs as measured on single-photon emission computerized tomography (SPECT), a type of metabolic imaging that shows increased regional blood flow (RBF) during ictal activity. ${ }^{67,68}$

SIRPIDs can often be observed in patients who have poor outcomes, particularly in postcardiac arrest patients when seen during therapeutic hypothermia in a small prospective study. ${ }^{69}$ Despite this, multiple studies have shown that SIRPIDs are not independently associated with increased in-hospital mortality or outcomes at discharge, ${ }^{61,64}$ suggesting that any increased morbidity or mortality seen with SIRPIDs can be attributable to their underlying etiology or their association with NCSE.

\section{When (and If) to Treat?}

No clear guidelines exist regarding if, when, or how best to treat the various epileptiform patterns that lie on the IIC. Seizures, and particularly status epilepticus, have been shown to cause neuronal injury and lead to increased risk of mortality, ${ }^{5-7,10}$ but to what degree this correlation can be extrapolated to IIC patterns is extremely difficult to determine with EEG alone. Some advocate the use of surrogate imaging, invasive multimodal monitoring, or serum markers in combination with EEG to attempt to identify the potential for neuronal injury and guide treatment decisions. ${ }^{48,50,68,70}$ Others advocate for empirically treating with antiseizure medications, as many of these patterns themselves carry 
high rates of morbidity and mortality, aside from their association with increased seizure risk. ${ }^{71,72}$ We will discuss these approaches here and present our treatment algorithm (-Fig. 5).

\section{Imaging}

Perhaps the most commonly described imaging modality used for this purpose is diffusion-weighted imaging (DWI) sequence MRI. Multiple studies have shown periictal diffusion restriction, typically in the thalamus and hippocampus, and particularly with generalized and complex partial status epilepticus. $^{73-76}$ These DWI changes are hypothesized to represent increased metabolic demand and potentially neuronal swelling. ${ }^{74}$ Some advocate for using presence or absence of DWI changes during IIC patterns to help predict neuronal injury and guide the decision to treat, but limited

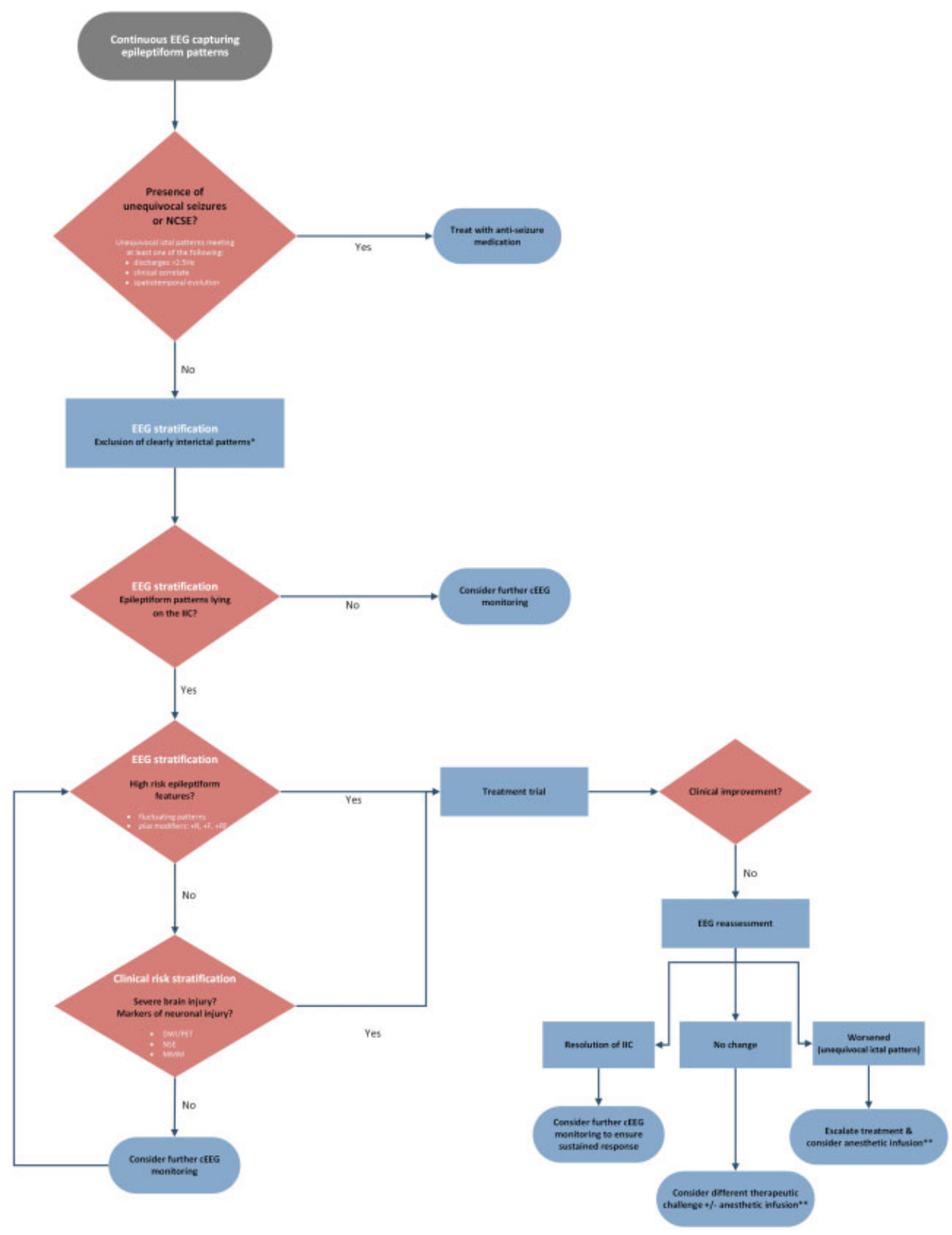

Fig. 5 A clinical approach to the ictal-interictal continuum. "If EEG findings are interictal, no need for a medication trial. The duration of monitoring is at the discretion of the treatment team, as depending on associated risk factors, such as level of consciousness and history of seizures, monitoring for $>24$ hours may be of highest yield for excluding nonconvulsive seizures. ${ }^{* *}$ Given the potential associated morbidity of anesthetic infusions for the treatment of refractory patterns lying on the IIC, these treatments are often reserved for unequivocally ictal patterns, such as NCSE. Nonetheless, in selected cases, these may be considered as a last therapeutic resort. cEEG, continuous electroencephalography; DWI, diffusion-weighted imaging; EEG, electroencephalography; MMM, multimodal monitoring; NCSE, nonconvulsive status epilepticus; PET, positron emission tomography. 
data exist on this application. Interestingly, one small observational study found that of 10 patients with LPDs, DWI changes were seen only in the 5 patients, all of whom also had seizures, suggesting that LPDs do not cause the same degree of neuronal swelling as seizures. ${ }^{77}$ SPECT has been studied in an attempt to answer this question. Some reports have, indeed, shown increased RBF during LPDs and corresponding normalization of RBF with the resolution of LPDs. ${ }^{50,78-80}{ }^{18} \mathrm{~F}$-fluorodeoxyglucose positron emission tomography, a modality that measures glucose uptake and typically shows increased uptake during seizures, has also been studied in LPDs with similar results. Several authors have reported cases in which patients demonstrated hypermetabolism during LPDs with resolution of hypermetabolism when LPDs resolve. ${ }^{78,81}$

\section{Serum Markers and Multimodal Monitoring}

Emerging data suggest that various serum and cerebrospinal fluid biomarkers, as well as invasive monitoring data, may serve as valuable surrogate markers of neuronal injury. Neuron-specific enolase (NSE) is a substance contained within neurons, and thus its detection in serum can be used as a marker of neuronal injury and breakdown of the blood-brain barrier. ${ }^{82}$ It has been shown that NSE is elevated in patients with multiple types of status epilepticus (including complex partial, absence, convulsive, and myoclonic), but more so in complex partial and myoclonic status. ${ }^{70}$ While NSE may be helpful in guiding treatment of IIC rhythms, interpretation of elevated levels must be done with caution. NSE is also present in neuroendocrine tissues, erythrocytes, ${ }^{83}$ and platelets, ${ }^{84}$ potentially leading to falsely elevated levels in neuroendocrine tumors or hemolyzed samples.

Intracranial EEG (depth EEG or dEEG, and electrocorticography) and microdialysis are being increasingly used to monitor seizure activity potentially missed on scalp EEG as well as various metabolic parameters in critically ill patients. Multiple studies have shown that intracranial EEG often detects seizures and PDs that go undetected on scalp EEG, suggesting a higher sensitivity and greater potential for earlier intervention. In one study of 34 patients with severe TBI, $61 \%$ of patients had NCSz or PDs, and of these, $42.9 \%$ were captured only on dEEG. ${ }^{85}$ In another group of 48 patients with high-grade SAH, $38 \%$ had seizures on dEEG, whereas only $8 \%$ of these were also detected on scalp EEG, and similar findings were seen in a related study of patients with highgrade SAH. ${ }^{12,86}$ Although patients in these studies all had primary neurological injuries as the indication for invasive monitoring with intracranial EEG, it is reasonable to suspect that other critically ill patients without primary neurological injury who have unexplained coma may indeed be having NCSz or PDs occurring at a level that is not detected by scalp EEG alone.

These latter two studies in patients with aSAH also examined increases in regional cerebral blood flow (rCBF) and partial pressure of oxygen in interstitial brain tissue $\left(\mathrm{PbO}_{2}\right)$ during seizures and PDs. In the first study, periictal increases in $\mathrm{rCBF}$ and decreases in $\mathrm{Pbo}_{2}$ did not reach statistical significance. ${ }^{12}$ In the second, however, there appeared to be a statistically significant decline in $\mathrm{Pbo}_{2}$ time-locked to PDs with frequencies $>2 \mathrm{~Hz}$ beginning 5 to 10 minutes after onset of discharges. ${ }^{86}$ They also demonstrated a rise in $\mathrm{rCBF}$ with PDs as seen in seizures but found that at frequencies $>2.5 \mathrm{~Hz}$ there was a relative decline in cerebral perfusion pressures (i.e., $97 \mathrm{~mm} \mathrm{Hg}$ during $2 \mathrm{~Hz}$ PDs decreasing to $95 \mathrm{~mm} \mathrm{Hg}$ for $2.5 \mathrm{~Hz}$ PDs and $67.8 \mathrm{~mm} \mathrm{Hg}$ for $3 \mathrm{~Hz}$ PDs $){ }^{86}$ These data suggest that higher frequency PDs may be associated with brain tissue hypoxia and inadequate rCBF to compensate for increased metabolic demand (e.g., neurovascular coupling and cerebral autoregulation), thus potentially arguing for the treatment of IIC patterns to prevent secondary brain injury.

\section{Empiric Treatment}

Historically, empiric treatment trials with low-dose benzodiazepines for IIC patterns were considered positive when both an electrographic and clinical improvement was observed. ${ }^{14,66,71,87}$ Unfortunately, these trials are often equivocal with apparent electrographic improvement without corresponding clinical improvement, in part attributed to poor baseline mental status in critical illness and compounded by the sedative effect of benzodiazepines. ${ }^{50,72,88}$ Furthermore, one retrospective study showed no clear benefit and increased mortality in elderly patients with NCSE treated with benzodiazepines. ${ }^{89}$ Some centers have instead advocated a trial of a nonsedating ASD over a benzodiazepine as an initial choice to better assess for clinical improvement in the absence of iatrogenic sedation. ${ }^{87,90}$ Less sedating ASDs that have been used include IV fosphenytoin, valproate, as well as both IV and oral levetiracetam ${ }^{91}$ and oral topiramate. ${ }^{92}$ With specific regard to SIRPIDs, it is unclear if the appropriate management involves using ASDs, minimizing stimulation or administering bolus benzodiazepines before necessary stimulation.

\section{Conclusions}

Over the last decade, the increased use of cEEG monitoring in the critical care setting has allowed detection of potentially malignant patterns that would have been otherwise missed on routine $\mathrm{EEG},{ }^{5}$ facilitated earlier detection and initiation of treatment of NCSz and NCSE, and provided valuable diagnostic and prognostic information in a variety of clinical scenarios. However, the acquisition of large amounts of electrophysiological data in critically ill patients has also resulted in increased detection of EEG patterns whose clinical significance remains unclear, patterns that are neither interictal nor definitively ictal. Although there are increasing scientific efforts aimed at further characterizing these patterns, their outcomes and their potential for neuronal injury, there remain no standardized guidelines for management. There is compelling evidence that some patterns are more highly associated with seizures and may, in fact, be causing a similar degree of neuronal injury as seizures, thus warranting aggressive treatment. It is becoming more evident that these patterns differ etiologically and that the "best" treatment may not be ASDs, but rather blood pressure 
augmentation or spasmolysis in SAH, antibiotics or increased cerebral perfusion pressure in sepsis, or the institution of continuous venovenous hemofiltration and ECMO, for example, in organ failure. We have proposed one possible approach to these patterns, but there is no doubt that such algorithms will continue to evolve as etiology-specific therapeutic strategies are better defined. Further prospective studies using invasive and noninvasive multimodal monitoring are needed to validate existing surrogate biomarkers of neuronal injury and their correlation with ASD trials and changes in the physiologic and serologic milieu. By tailoring our therapeutic approach to our understanding of which patterns truly warrant "treatment" and with which intervention, we can begin to deliver sophisticated, brain-focused precision medicine that will prevent not only secondary injury but also improve functional and neurological outcomes for critically ill patients.

\section{Disclosure}

None.

\section{Funding}

None.

\section{References}

1 Abend NS, Dlugos DJ, Hahn CD, Hirsch LJ, Herman ST. Use of EEG monitoring and management of non-convulsive seizures in critically ill patients: a survey of neurologists. Neurocrit Care 2010; 12(03):382-389

2 Herman ST, Abend NS, Bleck TP, et al; Critical Care Continuous EEG Task Force of the American Clinical Neurophysiology Society. Consensus statement on continuous EEG in critically ill adults and children, part I: indications. J Clin Neurophysiol 2015;32(02): 87-95

3 Claassen J, Taccone FS, Horn P, Holtkamp M, Stocchetti N, Oddo M; Neurointensive Care Section of the European Society of Intensive Care Medicine. Recommendations on the use of EEG monitoring in critically ill patients: consensus statement from the neurointensive care section of the ESICM. Intensive Care Med 2013;39 (08):1337-1351

4 Brophy GM, Bell R, Claassen J, et al; Neurocritical Care Society Status Epilepticus Guideline Writing Committee. Guidelines for the evaluation and management of status epilepticus. Neurocrit Care 2012;17(01):3-23

5 Pandian JD, Cascino GD, So EL, Manno E, Fulgham JR. Digital videoelectroencephalographic monitoring in the neurological-neurosurgical intensive care unit: clinical features and outcome. Arch Neurol 2004;61(07):1090-1094

6 De Marchis GM, Pugin D, Meyers E, et al. Seizure burden in subarachnoid hemorrhage associated with functional and cognitive outcome. Neurology 2016;86(03):253-260

7 Payne ET, Zhao XY, Frndova H, et al. Seizure burden is independently associated with short term outcome in critically ill children. Brain 2014;137(Pt 5):1429-1438

8 Wagenman KL, Blake TP, Sanchez SM, et al. Electrographic status epilepticus and long-term outcome in critically ill children. Neurology 2014;82(05):396-404

9 Rush B, Wiskar K, Fruhstorfer C, Hertz P. Association between seizures and mortality in patients with aneurysmal subarachnoid hemorrhage: A nationwide retrospective cohort analysis. Seizure 2016;41:66-69

10 Punia V, Garcia CG, Hantus S. Incidence of recurrent seizures following hospital discharge in patients with LPDs (PLEDs) and nonconvulsive seizures recorded on continuous EEG in the critical care setting. Epilepsy Behav 2015;49:250-254

11 Claassen J, Mayer SA, Kowalski RG, Emerson RG, Hirsch LJ. Detection of electrographic seizures with continuous EEG monitoring in critically ill patients. Neurology 2004;62(10):1743-1748

12 Claassen J, Perotte A, Albers D, et al. Nonconvulsive seizures after subarachnoid hemorrhage: Multimodal detection and outcomes. Ann Neurol 2013;74(01):53-64

13 Hirsch LJ, LaRoche SM, Gaspard N, et al. American Clinical Neurophysiology Society's Standardized Critical Care EEG Terminology: 2012 version. J Clin Neurophysiol 2013;30(01):1-27

14 Chong DJ, Hirsch LJ. Which EEG patterns warrant treatment in the critically ill? Reviewing the evidence for treatment of periodic epileptiform discharges and related patterns. J Clin Neurophysiol 2005;22(02):79-91

15 Gaspard N, Hirsch LJ, LaRoche SM, Hahn CD, Westover MB; Critical Care EEG Monitoring Research Consortium. Interrater agreement for critical care EEG terminology. Epilepsia 2014;55(09):1366-1373

16 Foreman B, Mahulikar A, Tadi P, et al; Critical Care EEG Monitoring Research Consortium (CCEMRC). Generalized periodic discharges and 'triphasic waves': A blinded evaluation of inter-rater agreement and clinical significance. Clin Neurophysiol 2016;127(02): 1073-1080

17 Beniczky S, Hirsch LJ, Kaplan PW, et al. Unified EEG terminology and criteria for nonconvulsive status epilepticus. Epilepsia 2013; 54(Suppl 6):28-29

18 Leitinger M, Beniczky S, Rohracher A, et al. Salzburg consensus criteria for non-convulsive status epilepticus-approach to clinical application. Epilepsy Behav 2015;49:158-163

19 Hirsch LJ. Classification of EEG patterns in patients with impaired consciousness. Epilepsia 2011;52(Suppl 8):21-24

20 Newey CR, Sahota P, Hantus S. Electrographic features of lateralized periodic discharges stratify risk in the interictal-ictal continuum. J Clin Neurophysiol 2017;34(04):365-369

21 Pohlmann-Eden B, Hoch DB, Cochius JI, Chiappa KH. Periodic lateralized epileptiform discharges-a critical review. J Clin Neurophysiol 1996;13(06):519-530

22 Fitzpatrick W, Lowry N. PLEDs: clinical correlates. Can J Neurol Sci 2007;34(04):443-450

23 Lee JW, LaRoche S, Choi H, et al; Critical Care EEG Monitoring Research Consortium (CCEMRC). Development and feasibility testing of a critical care EEG monitoring database for standardized clinical reporting and multicenter collaborative research. J Clin Neurophysiol 2016;33(02):133-140

24 Sen-Gupta I, Schuele SU, Macken MP, Kwasny MJ, Gerard EE. "Ictal" lateralized periodic discharges. Epilepsy Behav 2014; 36:165-170

25 Swisher CB, Shah D, Sinha SR, Husain AM. Baseline EEG pattern on continuous ICU EEG monitoring and incidence of seizures. J Clin Neurophysiol 2015;32(02):147-151

26 Gaspard N, Manganas L, Rampal N, Petroff OA, Hirsch LJ. Similarity of lateralized rhythmic delta activity to periodic lateralized epileptiform discharges in critically ill patients. JAMA Neurol 2013;70(10):1288-1295

27 Orta DS, Chiappa KH, Quiroz AZ, Costello DJ, Cole AJ. Prognostic implications of periodic epileptiform discharges. Arch Neurol 2009;66(08):985-991

28 Gurer G, Yemisci M, Saygi S, Ciger A. Structural lesions in periodic lateralized epileptiform discharges (PLEDs). Clin EEG Neurosci 2004;35(02):88-93

29 San-Juan OD, Chiappa KH, Costello DJ, Cole AJ. Periodic epileptiform discharges in hypoxic encephalopathy: BiPLEDs and GPEDs as a poor prognosis for survival. Seizure 2009;18(05):365-368

30 Walsh JM, Brenner RP. Periodic lateralized epileptiform dischargeslong-term outcome in adults. Epilepsia 1987;28(05):533-536

31 Li HT, Wu T, Lin WR, et al. Clinical correlation and prognostic implication of periodic EEG patterns: A cohort study. Epilepsy Res 2017;131:44-50 
32 Claassen J, Jetté N, Chum F, et al. Electrographic seizures and periodic discharges after intracerebral hemorrhage. Neurology 2007;69(13):1356-1365

33 García-Morales I, García MT, Galán-Dávila L, et al. Periodic lateralized epileptiform discharges: etiology, clinical aspects, seizures, and evolution in 130 patients. J Clin Neurophysiol 2002;19(02): 172-177

34 Hartings JA, Williams AJ, Tortella FC. Occurrence of nonconvulsive seizures, periodic epileptiform discharges, and intermittent rhythmic delta activity in rat focal ischemia. Exp Neurol 2003; 179(02):139-149

35 Gross DW, Quesney LF, Sadikot AF. Chronic periodic lateralized epileptiform discharges during sleep in a patient with caudate nucleus atrophy: insights into the anatomical circuitry of PLEDs. Electroencephalogr Clin Neurophysiol 1998;107(06): 434-438

36 Chatrian GE, Shaw CM, Leffman H. The significance of periodic lateralized epileptiform discharges in EEG: an electrographic, clinical and pathological study. Electroencephalogr Clin Neurophysiol 1964;17:177-193

37 Téllez-Zenteno JF, Pillai SN, Hill MD, Pillay N. Chronic PLEDs with transitional rhythmic discharges (PLEDs-plus) in remote stroke. Epileptic Disord 2007;9(02):164-169

38 de la Paz D, Brenner RP. Bilateral independent periodic lateralized epileptiform discharges. Clinical significance. Arch Neurol 1981; 38(11):713-715

39 Oddo M, Carrera E, Claassen J, Mayer SA, Hirsch LJ. Continuous electroencephalography in the medical intensive care unit. Crit Care Med 2009;37(06):2051-2056

40 Gilmore EJ, Gaspard N, Choi HA, et al. Acute brain failure in severe sepsis: a prospective study in the medical intensive care unit utilizing continuous EEG monitoring. Intensive Care Med 2015; 41(04):686-694

41 Kurtz P, Gaspard N, Wahl AS, et al. Continuous electroencephalography in a surgical intensive care unit. Intensive Care Med 2014; 40(02):228-234

42 Reiher J, Rivest J, Grand'Maison F, Leduc CP. Periodic lateralized epileptiform discharges with transitional rhythmic discharges: association with seizures. Electroencephalogr Clin Neurophysiol 1991;78(01):12-17

43 Rodriguez Ruiz A, Vlachy J, Lee JW, et al; Critical Care EEG Monitoring Research Consortium. Association of periodic and rhythmic electroencephalographic patterns with seizures in critically ill patients. JAMA Neurol 2017;74(02):181-188

$44 \mathrm{Kim}$ JA, Rosenthal ES, Biswal S, et al. Epileptiform abnormalities predict delayed cerebral ischemia in subarachnoid hemorrhage. Clin Neurophysiol 2017;128(06):1091-1099

45 Terzano MG, Parrino L, Mazzucchi A, Moretti G. Confusional states with periodic lateralized epileptiform discharges (PLEDs): a peculiar epileptic syndrome in the elderly. Epilepsia 1986; 27(04):446-457

46 Beaumanoir A, André-Obadia N, Nahory A, Zerbi D. Special types of periodic lateralized epileptiform discharges associated with confusional state in cerebral circulation insufficiency. Electroencephalogr Clin Neurophysiol 1996;99(03):287-292

47 Drury I, Klass DW, Westmoreland BF, Sharbrough FW. An acute syndrome with psychiatric symptoms and EEG abnormalities. Neurology 1985;35(06):911-914

48 Sivaraju A, Gilmore EJ. Understanding and managing the ictalinterictal continuum in neurocritical care. Curr Treat Options Neurol 2016;18(02):8

49 Sainju RK, Manganas LN, Gilmore EJ, et al. Clinical correlates and prognostic significance of lateralized periodic discharges in patients without acute or progressive brain injury: a case-control study. J Clin Neurophysiol 2015;32(06):495-500

50 Claassen J. How I treat patients with EEG patterns on the ictalinterictal continuum in the neuro ICU. Neurocrit Care 2009; 11(03):437-444
51 Carrera E, Claassen J, Oddo M, Emerson RG, Mayer SA, Hirsch LJ. Continuous electroencephalographic monitoring in critically ill patients with central nervous system infections. Arch Neurol 2008;65(12):1612-1618

52 Pedersen GL, Rasmussen SB, Gyllenborg J, Benedek K, Lauritzen M. Prognostic value of periodic electroencephalographic discharges for neurological patients with profound disturbances of consciousness. Clin Neurophysiol 2013;124(01):44-51

53 Foreman B, Claassen J, Abou Khaled K, et al. Generalized periodic discharges in the critically ill: a case-control study of 200 patients. Neurology 2012;79(19):1951-1960

54 Yemisci M, Gurer G, Saygi S, Ciger A. Generalised periodic epileptiform discharges: clinical features, neuroradiological evaluation and prognosis in 37 adult patients. Seizure 2003;12(07):465-472

55 Brenner RP, Schaul N. Periodic EEG patterns: classification, clinical correlation, and pathophysiology. J Clin Neurophysiol 1990; 7(02):249-267

56 Husain AM, Mebust KA, Radtke RA. Generalized periodic epileptiform discharges: etiologies, relationship to status epilepticus, and prognosis. J Clin Neurophysiol 1999;16(01):51-58

57 Sutter R, Stevens RD, Kaplan PW. Significance of triphasic waves in patients with acute encephalopathy: a nine-year cohort study. Clin Neurophysiol 2013;124(10):1952-1958

58 Faigle R, Sutter R, Kaplan PW. Electroencephalography of encephalopathy in patients with endocrine and metabolic disorders. J Clin Neurophysiol 2013;30(05):505-516

59 Kaplan PW, Sutter R. Affair with triphasic waves-their striking presence, mysterious significance, and cryptic origins: what are they? J Clin Neurophysiol 2015;32(05):401-405

60 Hirsch LJ, Claassen J, Mayer SA, Emerson RG. Stimulus-induced rhythmic, periodic, or ictal discharges (SIRPIDs): a common EEG phenomenon in the critically ill. Epilepsia 2004;45(02):109-123

61 Braksick SA, Burkholder DB, Tsetsou S, et al. Associated factors and prognostic implications of stimulus-induced rhythmic, periodic, or ictal discharges. JAMA Neurol 2016;73(05):585-590

62 Ong C, Gilmore E, Claassen J, Foreman B, Mayer SA. Impact of prolonged periodic epileptiform discharges on coma prognosis. Neurocrit Care 2012;17(01):39-44

63 Skjei KL, Kessler SK, Abend NS. Stimulus-induced rhythmic, periodic, or ictal discharges in a 13-year-old girl after an overdose and respiratory arrest. Pediatr Neurol 2011;45(05):350-351

64 Van Straten AF, Fesler JR, Hakimi R, Sheng T, Thompson DM, Hakimi AS. SIRPIDs: prevalence and outcome in critically ill patients. J Clin Neurophysiol 2014;31(05):418-421

65 Rossetti AO, Dunand M. Creutzfeldt-Jakob disease: evolution from nonconvulsive status epilepticus, through SIRPIDs, to generalized periodic discharges. Clin Neurophysiol 2007;118(11): 2533-2536

66 Kaplan PW, Duckworth J. Confusion and SIRPIDs regress with parenteral lorazepam. Epileptic Disord 2011;13(03):291-294

67 Smith CC, Tatum WO, Gupta V, Pooley RA, Freeman WD. SPECTnegative SIRPIDs: less aggressive neurointensive care? J Clin Neurophysiol 2014;31(03):e6-e10

68 Zeiler SR, Turtzo LC, Kaplan PW. SPECT-negative SIRPIDs argues against treatment as seizures. J Clin Neurophysiol 2011;28(05): 493-496

69 Alvarez V, Oddo M, Rossetti AO. Stimulus-induced rhythmic, periodic or ictal discharges (SIRPIDs) in comatose survivors of cardiac arrest: characteristics and prognostic value. Clin Neurophysiol 2013;124(01):204-208

70 DeGiorgio CM, Heck CN, Rabinowicz AL, Gott PS, Smith T, Correale J. Serum neuron-specific enolase in the major subtypes of status epilepticus. Neurology 1999;52(04):746-749

71 Lee JW. EEG in the ICU: what should one treat, what not? Epileptologie 2012;29:210-217

72 Rodríguez V, Rodden MF, LaRoche SM. Ictal-interictal continuum: A proposed treatment algorithm. Clin Neurophysiol 2016; 127(04):2056-2064 
73 Lansberg MG, O’Brien MW, Norbash AM, Moseley ME, Morrell M, Albers GW. MRI abnormalities associated with partial status epilepticus. Neurology 1999;52(05):1021-1027

74 Chu K, Kang DW, Kim JY, Chang KH, Lee SK. Diffusion-weighted magnetic resonance imaging in nonconvulsive status epilepticus. Arch Neurol 2001;58(06):993-998

75 Kavuk I, Koeppen S, Agelink M, Dörfler A, Limmroth V, Diener HC. Transient MRI abnormalities associated with partial status epilepticus. J Neurol 2004;251(09):1156-1157

76 Szabo K, Poepel A, Pohlmann-Eden B, et al. Diffusion-weighted and perfusion MRI demonstrates parenchymal changes in complex partial status epilepticus. Brain 2005;128(Pt 6):1369-1376

77 Narayanan J. Can diffusion-weighted imaging be used as a tool to predict seizures in patients with PLEDS? Epileptic Disord 2016;18 (04):440-446

78 Handforth A, Cheng JT, Mandelkern MA, Treiman DM. Markedly increased mesiotemporal lobe metabolism in a case with PLEDs: further evidence that PLEDs are a manifestation of partial status epilepticus. Epilepsia 1994;35(04):876-881

79 Ergün EL, Salanci BV, Erbaş B, Saygi S. SPECT in periodic lateralized epileptiform discharges (PLEDs): a case report on PLEDs. Ann Nucl Med 2006;20(03):227-231

80 Bozkurt MF, Saygi S, Erbas B. SPECT in a patient with postictal PLEDs: is hyperperfusion evidence of electrical seizure? Clin Electroencephalogr 2002;33(04):171-173

81 Struck AF, Westover MB, Hall LT, Deck GM, Cole AJ, Rosenthal ES. Metabolic correlates of the ictal-interictal continuum: FDG-PET during continuous EEG. Neurocrit Care 2016;24(03):324-331

82 DeGiorgio CM, Correale JD, Gott PS, et al. Serum neuron-specific enolase in human status epilepticus. Neurology 1995;45(06): 1134-1137
83 Ramont L, Thoannes H, Volondat A, Chastang F, Millet MC, Maquart FX. Effects of hemolysis and storage condition on neuron-specific enolase (NSE) in cerebrospinal fluid and serum: implications in clinical practice. Clin Chem Lab Med 2005;43(11): 1215-1217

84 Marangos PJ, Campbell IC, Schmechel DE, Murphy DL, Goodwin FK. Blood platelets contain a neuron-specific enolase subunit. J Neurochem 1980;34(05):1254-1258

85 Vespa P, Tubi M, Claassen J, et al. Metabolic crisis occurs with seizures and periodic discharges after brain trauma. Ann Neurol 2016;79(04):579-590

86 Witsch J, Frey HP, Schmidt JM, et al. Electroencephalographic periodic discharges and frequency-dependent brain tissue hypoxia in acute brain injury. JAMA Neurol 2017;74(03):301-309

87 Jirsch J, Hirsch LJ. Nonconvulsive seizures: developing a rational approach to the diagnosis and management in the critically ill population. Clin Neurophysiol 2007;118(08):1660-1670

88 Fountain NB, Waldman WA. Effects of benzodiazepines on triphasic waves: implications for nonconvulsive status epilepticus. J Clin Neurophysiol 2001;18(04):345-352

89 Litt B, Wityk RJ, Hertz SH, et al. Nonconvulsive status epilepticus in the critically ill elderly. Epilepsia 1998;39(11):1194-1202

90 O'Rourke D, Chen PM, Gaspard N, et al. Response rates to anticonvulsant trials in patients with triphasic-wave EEG patterns of uncertain significance. Neurocrit Care 2016;24(02):233-239

91 Rossetti AO, Bromfield EB. Levetiracetam in the treatment of status epilepticus in adults: a study of 13 episodes. Eur Neurol 2005;54(01):34-38

92 Towne AR, Garnett LK, Waterhouse EJ, Morton LD, DeLorenzo RJ. The use of topiramate in refractory status epilepticus. Neurology 2003;60(02):332-334 